\title{
Prediction of hemodynamic changes towards PEEP titrations at different volemic levels using a minimal cardiovascular model
}

\author{
C. Starfinger ${ }^{1}$, J.G. Chase ${ }^{1}$, C.E. Hann ${ }^{1}$, G.M.Shaw ${ }^{2}$, P. Lambert ${ }^{3}$, \\ B.W. Smith ${ }^{4}$, E. Sloth ${ }^{3}$, A. Larsson ${ }^{3}$, S. Andreassen ${ }^{4}$, S. Rees ${ }^{4}$ \\ ${ }^{1}$ Centre for Bioengineering, University of Canterbury, Christchurch, New Zealand \\ 2 Department of Intensive Care Medicine, Christchurch Hospital, Christchurch, New Zealand \\ 3 Department of Anesthesia, Emergency and Intensive Care Medicine, North Jutland, \\ Ålborg Hospital - Århus University Hospitals, Denmark \\ ${ }^{4}$ Center for Model-based Medical Decision Support, Ålborg University, Denmark
}

February 25, 2008

\section{Abstract}

A cardiovascular system model and parameter identification method have previously been validated for porcine experiments of induced Pulmonary Embolism and positive end-expiratory pressure (PEEP) titrations, accurately tracking all the main hemodynamic trends. In this research, the model and parameter identification process are further validated by predicting the effect of intervention. An overall population-specific rule linking specific model parameters to increases in PEEP is formulated to predict the hemodynamic 
effects on arterial pressure, pulmonary artery pressure and stroke volume. Hemodynamic changes are predicted for an increase from 0 to $10 \mathrm{~cm} \mathrm{H}_{2} \mathrm{O}$ with median absolute percentage errors less than 7\% (systolic pressures) and $13 \%$ (stroke volume). For an increase from 10 to $20 \mathrm{~cm} \mathrm{H}_{2} \mathrm{O}$ median absolute percentage errors are less than 11\% (systolic pressures) and 17\% (stroke volume). These results validate the general applicability of such a rule, which is not pig-specific, but holds over for all analyzed pigs. This rule enables physiological simulation and prediction of patient response. Overall, the prediction accuracy achieved represents a further clinical validation of these models, methods and overall approach to cardiovascular diagnosis and therapy guidance.

Keywords: cardiovascular system, cardiac model, parameter identification, integral method, PEEP, hypovolemia

\section{Introduction}

Circulatory dysfunctions and disease account for a significant number of ICU admissions. For example, a recent study found that $58 \%$ of ICU admissions for patients aged 65-74 years in Olmsted County, Minnesota were due to cardiovascular dysfunction, with a further $17 \%$ due to respiratory disease [1]. 
The treatment and management of this large group of patients is significantly affected by the difficulty in monitoring and managing circulatory status and affectivity.

However, cardiac disease states are highly patient-specific, such that every patient has a unique expression of the disease or dysfunction. They are thus difficult to accurately diagnose given the sometimes limited measurements available and the body's natural reflex responses to restore circulatory equilibrium, both of which can mask the underlying symptoms. Clinical staff must therefore consider many combinations of different disease scenarios based on frequently conflicting patient data, including clinical history and non-invasive and/or invasive studies [2]. Hence, successful diagnosis and treatment often rely on the experience and intuition of clinical staff, increasing the likelihood for clinical error, which is common with rates up to $50 \%$ $[3,4]$.

Tools for diagnosis and guiding therapy can help reduce variation and provide a more consistent care. Computerized protocols have thus become more widespread as they can be applied to complex clinical problems to create patient-specific therapy instructions $[5,6]$. A cardiovascular (CVS) model could be integrated into the clinical decision making process by offering 
clinicians the possibility of not only assisting in diagnosing, but also providing a model-based means to test different therapeutic procedures and their likely effect on the patient. Hence, treatment could be optimized for each patient and unnecessary interventions avoided.

A previously published integral-based parameter identification method [7] has been shown to successively identify pig-specific parameters for a minimal cardiac model [8]. These models and methods were further validated using a porcine experiment of PEEP titrations at different volemic levels [9], where the model's ability to capture the impact of pressure-volume changes with positive end-expiratory pressure (PEEP) and fluid therapy was shown.

PEEP is an operator-controlled variable that can be set during mechanical ventilation. The correct value of PEEP can be beneficial or detrimental for the patient dependent on how it is used. More specifically, in patients with lung injury, high PEEP levels may be necessary to maintain or restore oxygenation and for each individual patient the right balance between too much and too little PEEP has to be found. Too little PEEP may result in airway or alveolar collapse, whereas too much PEEP can cause alveolar overdistention and hemodynamic problems such as a reduction in cardiac output (CO). 
This research is a further validation of the overall diagnostic monitoring approach and extends the previously described methods. It illustrates one method for using this CVS model and integral-based parameter identification for therapy guidance and decision support by forward simulating the expected patient response to different interventions. Thus, this research provides a predictive validation of the model's capability and efficiency in a decision support role, rather than a physiological data matching validation. More specifically, general rules are developed relating to specific model parameters such that the model can then be used to predict the general patient response to increases in PEEP from 0 to $10 \mathrm{~cm} \mathrm{H}_{2} \mathrm{O}$ and from 10 to $20 \mathrm{~cm} \mathrm{H}_{2} \mathrm{O}$ during different volemic states.

\section{Methodology}

\section{$2.1 \quad$ CVS model}

The CVS model employed is a lumped parameter model based on earlier work $[8,10-13]$. This original model consisted of six elastic chambers, including two active chambers for the left and right ventricles. These pressure-volume chambers are each characterized by the flow in and out of the chamber, the pressure up- and downstream, the resistances of the heart valves, and inertia 
of the blood.

This original model has been extended and an overview of the new, extended model is given in Figure 1. The new model includes one compartment for the lung capillaries and a second for the body capillaries, thus separating the venous and arterial systems and resistances. This differentiation is critical when examining heart-lung interactions during positive pressure ventilation (PPV), especially when considering the application of different PEEP levels.

More specifically, increases in intrathoracic pressure $\left(P_{t h}\right)$ due to PEEP cause right ventricular preload to decrease by increasing the resistance to venous return $\left(R_{v r}\right)$. As a result, left ventricular afterload also decreases during PEEP as the pressure on the surface of the left ventricle is increased $[14,15]$. This LV afterload reduction is represented in the model as a decrease in systemic resistance $\left(R_{\text {sys }}\right)$. One can see, that two resistances on either side, the arterial and venous side, are necessary to correctly simulate the complex behaviors clinically observed during mechanical or spontaneous breathing. Note also that the original models use of a single resistor would not be able to accurately capture or predict this behaviour [11]. 


\subsection{Volume Calculations}

The parameter identification process uses the two ventricle volumes as input signals to accurately determine some of the parameters [7]. More specifically, the end-diastolic and end-systolic ventricle volumes (EDV, ESV) are needed. However, these volume measurements are usually not available in a clinical environment and thus need to be estimated from the readily available data. Currently, the LVEDV and RVEDV are estimated based on an estimated total blood volume (TBV) and the measured global end-diastolic volume (GEDV). As the stroke volume (SV) is a measured variable, ESV can be calculated by subtracting SV from EDV.

The total blood volume is estimated as $85 \mathrm{ml} / \mathrm{kg}$, with $25 \mathrm{ml} / \mathrm{kg}$ being stressed volume and $60 \mathrm{ml} / \mathrm{kg}$ unstressed volume [16, 17]. GEDV is the total end-diastolic volume of the left and right ventricle and the two atria, and is directly measured using the PiCCO monitor (Pulsion Medical Systems, Munich, Germany). The remaining model volumes are estimated based on known blood distributions $[18,19]$. Importantly, the volume in the pulmonary capillary and vein compartments $\left(V_{c a p}, V_{p v}\right)$ are given by the pulmonary blood volume (PBV) which is approximated as GEDV/4 [20]. The volumes in the aorta and pulmonary artery are also directly given, as the 
pressures $P_{a o}$ and $P_{p a}$ are measured and the elastances $E_{a o}$ and $E_{p a}$ are precalculated and remain fixed during the identification process. More details of the general identification process can be found in [9].

\subsection{Parameter Identification}

Model parameters are identified based on a previously described integralbased parameter identification method $[7,8]$. Briefly, to uniquely determine the parameters, the model equations are transformed using integrals. The integral-based parameter identification method is extended to rapidly identify the patient specific parameters from limited discrete data. The assumed measured or estimated data are the:

- discrete minimum and maximum values of the pressure in the aorta $\left(P_{a o, \max }, P_{a o, \min }\right)$

- discrete minimum and maximum values of the pressure in pulmonary $\operatorname{artery}\left(P_{p a, \max }, P_{p a, \min }\right)$

- mean central venous pressure $\left(C V P_{\text {mean }}\right)$

- discrete maximum and minimum volumes of the left and right ventricles $\left(V_{l v, \text { max }}, V_{l v, \min }, V_{r v, \max }, V_{r v, \min }\right)$ 
As the waveforms are not known, the integral method of [7] cannot be directly applied. However, waveforms can be artificially generated by scaling a set of previously calculated model outputs to best fit the maximal and minimal measured data values for the pressures and volumes. The assumption is that these waveforms are reasonably conformable with the actual clinical case based on prior physiological model validation studies $[8,11,13]$.

These scaled waveforms are then re-identified and a new CVS forward simulation is performed with the newly identified parameters. This simulated output is then compared to the clinical data. Subsequently, the output signals are re-scaled and a further set of new parameters are identified and used to run another simulation. This iterative process is stopped when the relative error between model output and clinical data reaches a set tolerance. More details can be found in [7-9].

\subsection{Prediction process and PEEP-specific model pa- rameters}

As shown previously, the CVS model parameters can be obtained accurately and repeatably for the porcine experiment of PEEP titrations [9]. Furthermore, very good correlations were found between specific model parameters and specific output signals [9]. These initial good results allow the assump- 
tion that an overall rule can be created that links changes in PEEP to corresponding changes in specific CVS model parameters. A general rule that is true for all studied pigs would allow the implementation of PEEP-specific parameters that change according to the currently applied PEEP. Such PEEPvarying parameters would enable more realistic and physiologically correct simulation of the cardiovascular system with the potential for using such forward simulations for diagnosis and therapy decision support.

The rules for PEEP-specific parameters are obtained by examining the percentage changes in the CVS model parameters for pig 1 during the different PEEP settings and volemic levels. As it is assumed, that the CVS model parameters are identified reliably, it can be expected that the rules obtained from only one pig also hold true for predicting the response of the remaining pigs. These parameter changes can however also be explained by reflecting on the known physiological effects of mechanical ventilation and especially PEEP on the circulation. Specifically, during the application of positive pressure ventilation (PPV) with PEEP, intrathoracic pressure increases and venous return is decreased. This decrease occurs not by altering the pressure gradient $\left(P_{s y s}-P_{r a}\right)$, but by increasing the resistance to venous return $R_{v r}[14,15]$. Hence, different PEEP levels should result in different values 
for $R_{v r}$. More specifically, $R_{v r}$ should increase during elevated PEEP levels. Furthermore, it is also known that right ventricular afterload increases during PPV [14, 15], so one would expect $R_{\text {pulin }}$ to also increase with increasing levels of PEEP.

\subsection{PEEP experiment study protocol}

\subsubsection{Instrumentation, Monitoring and Interventions}

The experiment was approved by the Danish National Animal Ethics Committee and data from six 20-22 kg pigs was analyzed for this research. Note, that the data obtained from 1 pig were not included in the study, as this pig had corrupted arterial pressure measurements for some volemic states, which prevented a reasonable prediction for this particular pig. A detailed description of the anesthesia, ventilation, instrumentation, monitoring procedure and performed interventions has been published in [21] and a brief overview of the study protocol and interventions is also given in [9].

\section{Results}

\subsection{PEEP-specific changes in resistances and volumes}

Table 1 shows how the CVS model identified parameters are affected by changes in PEEP from 0 to $10 \mathrm{~cm} \mathrm{H}_{2} \mathrm{O}$ and from 10 to $20 \mathrm{~cm} \mathrm{H}_{2} \mathrm{O}$. The per- 
centage changes represent values obtained from pig 1 as studied as detailed in [9]. The CVS model volumes are calculated for PEEP $0 \mathrm{~cm} \mathrm{H}_{2} \mathrm{O}$ as described previously [9]. Table 2 shows how the volumes are then adjusted for predicting the volume changes for PEEP of 10 and $20 \mathrm{~cm} \mathrm{H}_{2} \mathrm{O}$, respectively. These values were obtained by observing the model identified volume changes, but could also be explained by expected physiological PEEP-induced changes $[16,22]$ and direct examination of the data.

\subsection{Prediction of arterial pressures and stroke volume}

Predictions are made using data identified at PEEP of $0 \mathrm{~cm} \mathrm{H}_{2} \mathrm{O}$ for each pig. The estimated PEEP-specific parameter and volume changes in Tables 1 and 2 are used to predict changes in a given pigs model parameters and initial volume conditions. The modified PEEP-specific parameters are then used to simulate the therapy intervention with results compared to the clinical data.

Figure 2 shows the prediction results obtained for all pigs. In the upper panel, the predicted stroke volume (SV) is displayed with a cross, whereas the measured clinical values are shown as a dotted line. Predictions were made for PEEP values of 10 and $20 \mathrm{~cm} \mathrm{H}_{2} \mathrm{O}$ for the different volemic levels

of normovolemia ( $\mathrm{N}$ and $\mathrm{I1}$ ), hypovolemia $(\mathrm{H})$ and the two infusion-induced 
hypervolemic states (I2 and I3). Note that the values for PEEP of $0 \mathrm{~cm} \mathrm{H}_{2} \mathrm{O}$ are not shown as no predictions were performed for these PEEP values. The middle panel shows the predicted (cross) versus clinical (dotted line) systolic arterial pressure values (SAP) and the lower panel shows the results for predicting systolic pulmonary artery pressure (SPAP). Table 3 summarizes the prediction results for all 6 pigs and gives the median and maximum absolute percentage errors and the interquartile range (IQR) for predicting stroke volume (SV), systolic arterial pressure (SAP) and systolic pulmonary artery pressure (SPAP).

\section{Discussion}

It is well known that PEEP often reduces venous return (VR) and thus cardiac output (CO). Recent publications suggest that the main effect by which PEEP decreases venous return is by increasing the resistance to venous return $\left(R_{v r}\right)[14,15]$. Hence, different PEEP levels should result in different values for $R_{v r}$ or more specifically, $R_{v r}$ should increase during elevated PEEP. Table 1 shows how $R_{v r}$ is increased with increasing PEEP values, matching physiological expectations. Furthermore, it is also known that right ventricular afterload increases during PPV $[14,15]$, so one would expect $R_{\text {pulin }}$ to also 
increase with increasing levels of PEEP. Analogously, the parameter changes for LV afterload $\left(R_{\text {sys }}\right)$ and $\mathrm{LV}$ preload $\left(R_{\text {pulout }}\right)$ can be derived.

All other parameter changes can be explained similarly. Thus, for example, increasing arterial elastance values $\left(E_{a o}\right.$ and $\left.E_{p a}\right)$ are observed during the PEEP titration experiment and are expected because the pulse pressure/stroke volume ratio (PP/SV) determines the arterial elastances and during PEEP, stroke volume decreases more than pulse pressure does. Venous elastances $\left(E_{v c}\right.$ and $\left.E_{p u}\right)$ are assumed to increase as well because positive endexpiratory pressure elevates the transpulmonary pressure which compresses the large intrathoracic veins and right atrium. Note however that the systemic elastance $\left(E_{\text {sys }}\right)$ remains constant as it has been shown that increases in PEEP decrease unstressed volume and thereby increase stressed volume with no change in compliance [23].

It can also be expected, that the pericardium becomes stiffer (diastolic elastance $P_{0 p c d}$ increases) with increasing levels of PEEP, as the heart becomes more and more compressed by the expanding lung and pressurized thoracic cavity. Similarly, $E_{c a p}$, the lung capillaries elastance is expected to increase with falling pulmonary blood volume (PBV) values and thus decreases in compliance. Note, that $R_{\text {sys }}$ remains constant during hypovolemia, 
assuming a compensation caused by an increased sympathetic activity in response to the blood loss. Table 1 shows these parameter variations matching clinical observations in their general trends.

Changes in the volumes can be similarly explained. As GEDV decreases with increasing PEEP levels, it can be followed that LVEDV and RVEDV have to decrease, as well. $V_{p v}$ and $V_{c a p}$ are expected to decrease as given by the drop in pulmonary blood volume. Note, that $V_{\text {sys }}$ remains constant as blood is shifted centrally to help maintain CVP. However, $V_{s y s}$ is simulated to slightly decrease during hypovolemia, as hypovolemia inhibits fluid reabsorption due to high capillary pressures [22]. Table 2 shows the general trend of these volume variations.

Figure 2 and Table 3 show how the CVS model and these PEEP-specific variable changes were able to predict the main trends in the clinically relevant systolic and diastolic arterial pressures (SAP, DAP), systolic and diastolic pulmonary artery pressures (SPAP, DPAP) and stroke volume (SV). Note, that starting from only one pig it has been shown that a general population rule, valid over all studied pigs, can be formulated and used to predict hemodynamic changes, thus showing that all pigs react along similar trends. This assumption makes sense as the pigs were healthy and no other diseases 
or hemodynamic instabilities were induced. Overall, the prediction error results are within clinical variation and close to measurement error in some cases. These results thus show the general applicability of this CVS model and methods to predictively capture the main hemodynamic trends due to this often applied intervention over a number of different pigs.

\subsection{Study Limitations}

It has to be mentioned that for this research all model volumes had to be estimated, based only on an estimated TBV and measured GEDV. This approximation naturally introduces a potentially significant degree of uncertainty and possible source of model error. As can be seen in Figure 2 the prediction results during normo- and hypovolemia are relatively good with median absolute percentage errors less than $6 \%$ for the arterial pressures and less than $10 \%$ for the stroke volume. However, the prediction results are not that good for the hypervolemia states, suggesting a model error. In particular, the approximated volumes and any error they contain may well be exacerbated in this case. Further research will thus need to be conducted to better model or estimate the different volume compartments, especially during hypervolemic states. 


\section{Conclusion}

The integral-based optimization led to the successful definition of PEEPspecific population parameters for a minimal cardiac model. These PEEPspecific population values were used to validate the predictive ability of the model for use in guiding this often used therapy. This further validation shows the ability of the model to adequately and realistically simulate the impact of pressure-volume changes with PEEP and fluid therapy. Moreover, such rules can be similarly derived and used to predict the response towards a variety of interventions, while errors or deviations from can point out developing disease states or hemodynamic instabilities.

\section{References}

[1] Edward G Seferian and Bekele Afessa. Demographic and clinical variation of adult intensive care unit utilization from a geographically defined population. Crit Care Med, 34(8):2113-2119, Aug 2006.

[2] A. Grenvik, S. M. Ayres, P. R. Holbrook, and W. C. Shoemaker, editors. Textbook of Critical Care. W.B. Saunders Company, 2nd edition, 1989.

[3] N. S. Abramson, K. S. Wald, A. N. Grenvik, D. Robinson, and J. V. 
Snyder. Adverse occurrences in intensive care units. JAMA, 244(14): 1582-1584, Oct 1980.

[4] Alan H. Morris. Rational use of computerized protocols in the intensive care unit. Critical Care, 5:249-254, 2001.

[5] Andreas Kortgen, Petra Niederprüm, and Michael Bauer. Implementation of an evidence-based "standard operating procedure" and outcome in septic shock. Crit Care Med, 34(4):943-949, Apr 2006.

[6] L. Leibovici, M. Paul, A. D. Nielsen, E. Tacconelli, and S. Andreassen. The treat project: decision support and prediction using causal probabilistic networks. International Journal of Antimicrobial Agents, In Press, Corrected Proof, 2007.

[7] C. E. Hann, J. G. Chase, and G. M. Shaw. Integral-based identification of patient specific parameters for a minimal cardiac model. Comput Methods Programs Biomed, 81(2):181-192, Feb 2006.

[8] C. Starfinger, C. E. Hann, J. G. Chase, T. Desaive, A. Ghuysen, and G. M. Shaw. Model-based cardiac diagnosis of pulmonary embolism. Comput Methods Programs Biomed, 87(1):46-60, Jul 2007. 
[9] C. Starfinger, J.G. Chase, C.E. Hann, G.M. Shaw, P. Lambert, B.W. Smith, E. Sloth, A. Larsson, S. Andreassen, and S. Rees. Model-based analysis of peep titrations during different volemic levels. Comput Methods Programs Biomed, 2008. in review.

[10] D. C. Chung, S. C. Niranjan, J. W. Clark JR, A. Bidani, W. E. Johnston, J. B. Zwischenberger, and D. L. Traber. A dynamic model of ventricular interaction and pericardial influence. Am. J. Physiol., 272(6 Pt 2):H2942-2962, 1997.

[11] B. W. Smith, J. G. Chase, R. I. Nokes, G. M. Shaw, and G. Wake. Minimal haemodynamic system model including ventricular interaction and valve dynamics. Medical Engineering 8 Physics, 26(2):131-139, 2004.

[12] C. E. Hann, J. G. Chase, and G. M. Shaw. Efficient implementation of non-linear valve law and ventricular interaction dynamics in the minimal cardiac model. Comput Methods Programs Biomed, 80(1):65-74, Oct 2005.

[13] Bram W Smith, J. Geoffrey Chase, Geoff M Shaw, and Roger I Nokes. 
Simulating transient ventricular interaction using a minimal cardiovascular system model. Physiol Meas, 27(2):165-179, Feb 2006.

[14] H.E. Fessler. Heart-lung interactions: applications in the critically ill. European Respiratory Journal, Series 'Clinical Physiology in Respiratory Intensive Care', 10(12):226-237, 1997.

[15] A.M. Miro and M.R. Pinsky. Pediatric Critical Care, chapter 24, pages 249-256. Mosby, 3 edition, 2005.

[16] C. F. Rothe. Reflex control of the veins in cardiovascular function. Physiologist, 22(2):28-35, Apr 1979.

[17] F. S. Grodins. Integrative cardiovascular physiology: a mathematical synthesis of cardiac and blood vessel hemodynamics. Q. Rev. Biol., 34: 93-116, 1959.

[18] R. E. Klabunde. Cardiovascular Physiology Concepts. Lippincott Williams and Wilkins, 2004.

[19] A.C. Guyton and J.E. Hall. Textbook of medical physiology. W.B. Saunders Company, Philadelphia, 10th edition, 2000. 
[20] Pulsion medical systems, munich germany: Picco plus user presentation, February 2005.

[21] P. Lambert, E. Sloth, B. Smith, L. K. Hansen, J. Koefoed-Nielsen, E. Tonnesen, and A. Larsson. Does a positive end-expiratory pressureinduced reduction in stroke volume indicate preload responsiveness? an experimental study. Acta Anaesthesiol Scand, 51(4):415-425, Apr 2007.

[22] J. Peters, G.W. Mack, and G. Lister. The importance of the peripheral circulation in critical illness. Intensive Care Medicine, 27:1446-1458, 2001.

[23] S. Nanas and S. Madger. Adaptations of the peripheral circulation to peep. Am Rev Respir Dis, 146:658-693, 1992. 


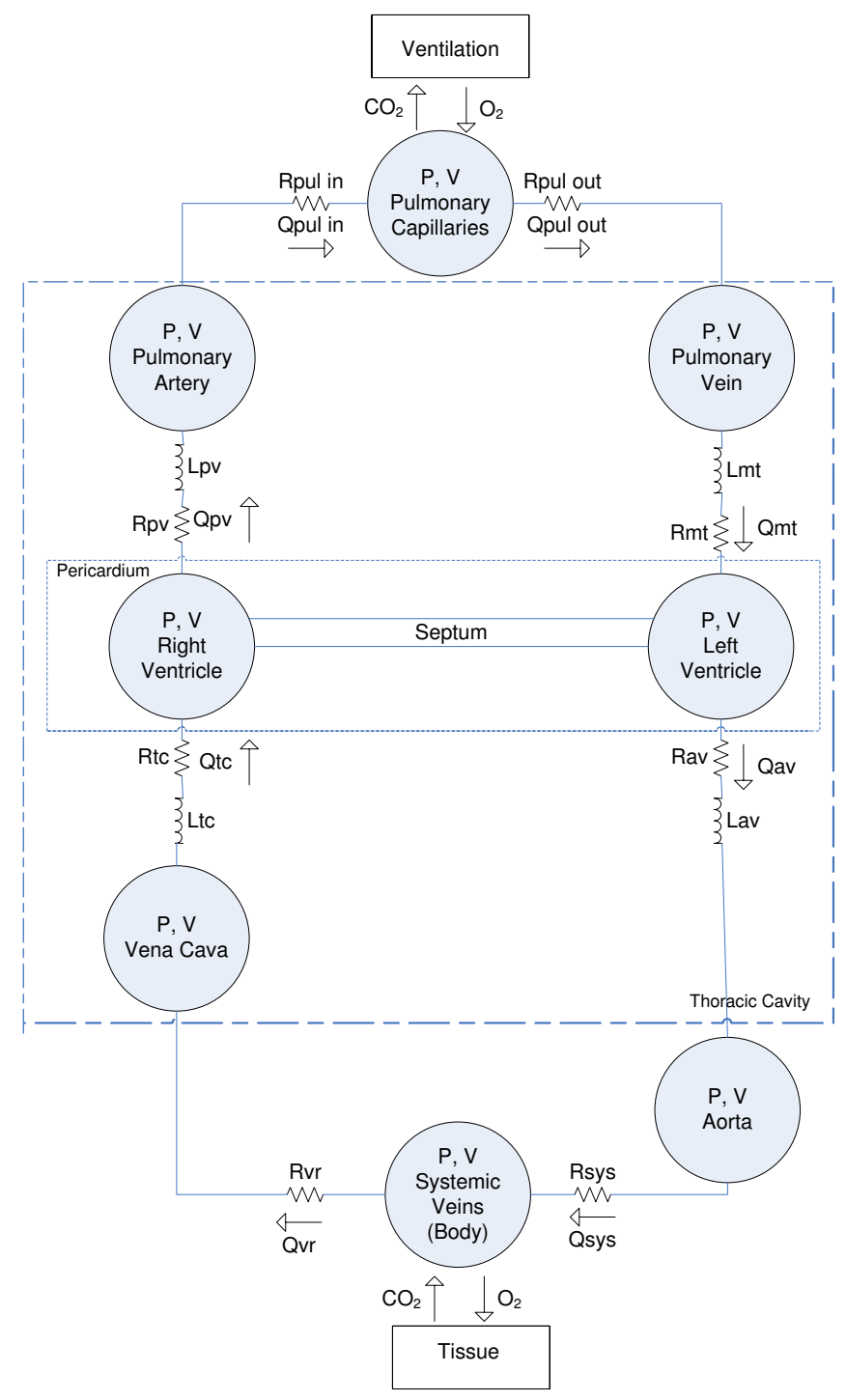

Figure 1: Extended CVS model overview which includes additional compartments $P, V_{\text {sys }}$ and $P, V_{\text {cap }}$ to differentiate the arterial and venous sides of the pulmonary and systemic circulation. 


\begin{tabular}{|l|l|l|}
\hline \multicolumn{3}{|l|}{ PEEP-specific parameter changes } \\
\hline Parameter & $0 \mathrm{~cm} \mathrm{H}_{2} \mathrm{O} \Rightarrow 10 \mathrm{cmH}_{2} \mathrm{O}$ & $10 \mathrm{~cm} \mathrm{H}_{2} \mathrm{O} \Rightarrow 20 \mathrm{~cm}_{2} \mathrm{O}$ \\
\hline$E_{a o}$ & plus $10 \%$ & plus $20 \%$ \\
$E_{p a}$ & plus $10 \%$ & plus $60 \%$ \\
$E_{v c}$ & plus $10 \%$ & plus $10 \%$ \\
$E_{\text {pu }}$ & plus $5 \%$ & plus $5 \%$ \\
$R_{\text {sys }}$ & minus $10 \%$ & minus $10 \%$ \\
$R_{\text {vr }}$ & plus $30 \%$ & plus $50 \%$ \\
$R_{\text {pulin }}$ & plus $40 \%$ & plus $60 \%$ \\
$R_{\text {pulout }}$ & plus $15 \%$ & plus $30 \%$ \\
$E_{\text {cap }}$ & plus $40 \%$ & plus $50 \%$ \\
$P_{\text {opcd }}$ & plus $10 \%$ & plus $10 \%$ \\
\hline
\end{tabular}

Table 1: Parameter changes for forward simulating changes in PEEP from 0 to $10 \mathrm{~cm} \mathrm{H}_{2} \mathrm{O}$ and from 10 to $20 \mathrm{~cm} \mathrm{H}_{2} \mathrm{O}$.

\begin{tabular}{|l|l|l|}
\hline \multicolumn{3}{|l|}{ PEEP-specific volume changes } \\
\hline Volume & $0 \mathrm{~cm}_{2} \mathrm{O} \Rightarrow 10 \mathrm{~cm}_{2} \mathrm{O}$ & $10 \mathrm{~cm} \mathrm{H}_{2} \mathrm{O} \Rightarrow 20 \mathrm{~cm}_{2} \mathrm{O}$ \\
\hline$V_{l v}$ & minus $25 \%$ & minus $25 \%$ \\
$V_{r v}$ & minus $20 \%$ & minus $20 \%$ \\
$V_{p v}$ & minus $30 \%$ & minus $30 \%$ \\
$V_{v c}$ & minus $30 \%$ & minus $50 \%$ \\
$V_{p a}$ & minus $10 \%$ & minus $35 \%$ \\
$V_{a o}$ & minus $25 \%$ & minus $50 \%$ \\
$V_{\text {sys }}$ & plus $2 \%$ & plus $2 \%$ \\
$V_{c a p}$ & minus $2 \%$ & minus $2 \%$ \\
\hline
\end{tabular}

Table 2: Volume changes for forward simulating changes in PEEP from 0 to $10 \mathrm{~cm} \mathrm{H}_{2} \mathrm{O}$ and from 10 to $20 \mathrm{~cm} \mathrm{H}_{2} \mathrm{O}$. 


\begin{tabular}{|l|l|l|l|l|l|}
\hline \multicolumn{7}{|l|}{ Prediction of PEEP-induced hemodynamic changes } \\
\hline All pigs, PEEP 10 & SAP & DAP & SPAP & DPAP & SV \\
\hline median & 6.65 & 9.80 & 4.24 & 5.94 & 12.24 \\
max & 22.62 & 31.89 & 12.92 & 18.71 & 38.95 \\
iqr & 7.64 & 11.14 & 3.78 & 5.52 & 13.05 \\
\hline All pigs, PEEP 20 & SAP & DAP & SPAP & DPAP & SV \\
\hline median & 10.47 & 12.99 & 6.51 & 7.14 & 16.86 \\
max & 30.69 & 33.82 & 12.82 & 18.24 & 33.61 \\
iqr & 13.26 & 14.03 & 6.98 & 5.25 & 13.08 \\
\hline \hline All pigs, all predictions & SAP & DAP & SPAP & DPAP & SV \\
\hline median & 7.93 & 11.52 & 4.62 & 7.02 & 14.49 \\
max & 30.69 & 33.82 & 12.92 & 18.71 & 38.95 \\
iqr & 8.10 & 13.86 & 5.54 & 8.40 & 12.58 \\
\hline
\end{tabular}

Table 3: Absolute median and maximum percentage error and interquartile range (iqr) for predicted values of SAP/DAP $=$ systolic/diastolic arterial pressure, $\mathrm{SPAP} / \mathrm{DPAP}=$ systolic/diastolic pulmonary artery pressure and $\mathrm{SV}=$ stroke volume. 

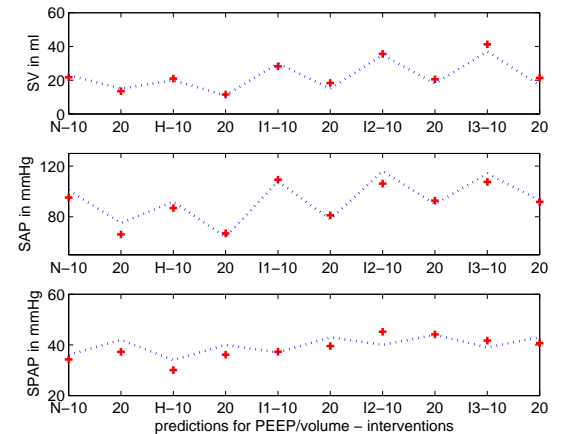

(a) pig 1
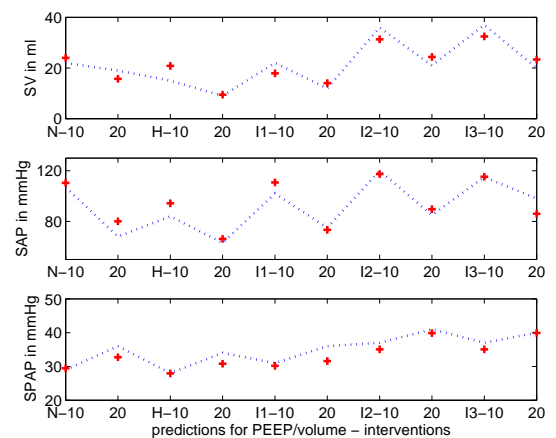

(c) pig 3
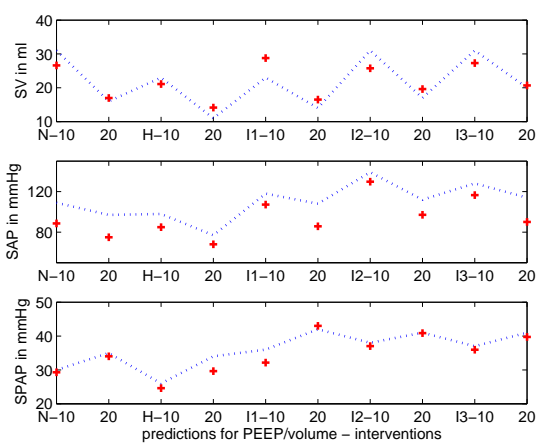

(e) $\operatorname{pig} 5$
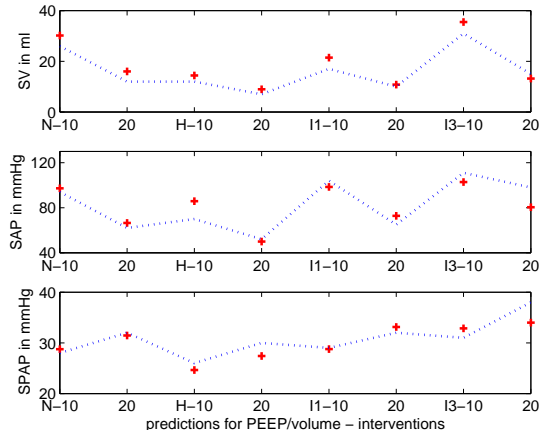

(b) pig 2
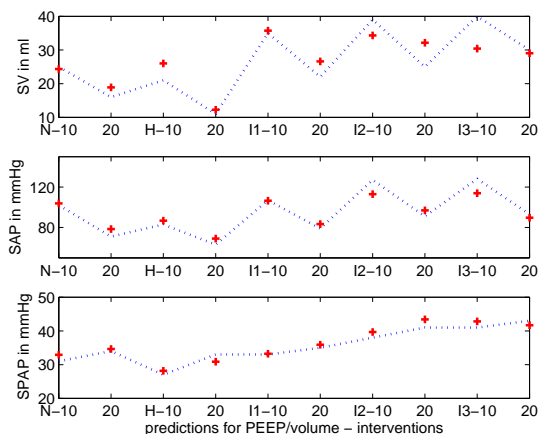

(d) pig 4
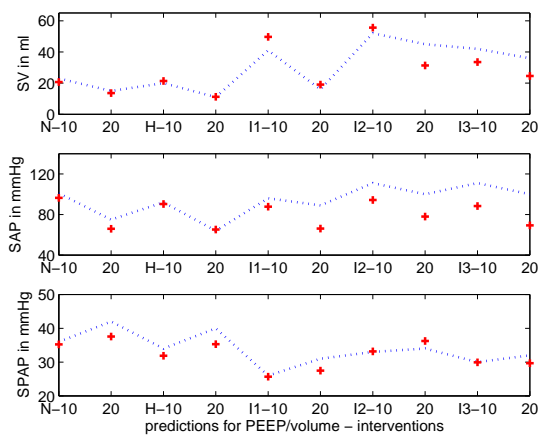

(f) $\operatorname{pig} 6$

Figure 2: Model prediction (cross) ys clinical (dotted line) pressures and volumes for pig 1-6 for PEEP 10 and $20 \mathrm{~cm} \mathrm{H}_{2} \mathrm{O}$. The upper panel shows the stroke volume (SV), the middle panel shows the systolic arterial pressure (SAP) and the lower panel shows the systolic pulmonary artery pressure (SPAP). 